\title{
Origin of water on the terrestial planets
}

\author{
Michael J. Drake ${ }^{1}$ and Humberto Campins ${ }^{2}$ \\ ${ }^{1}$ Lunar and Planetary Laboratory, University of Arizona, Tucson, AZ 85721, USA \\ email: drake@lpl.arizona.edu \\ ${ }^{2}$ Physics Department, University of Central Florida, Orlando, FL 32816, USA \\ email: campins@physics.ucf.edu
}

\begin{abstract}
We examine the origin of water in the terrestrial planets. We list various geochemical measurements that may be used to discriminate between different endogenous and exogenous sources of water. Late stage delivery of significant quantities of water from asteroidal and cometary sources appears to be ruled out by isotopic and molecular ratio considerations, unless either comets and asteroids currently sampled spectroscopically and by meteorites are unlike those falling to Earth 4.5 Ga ago or our measurements are not representative of those bodies. The dust in the accretion disk from which terrestrial planets formed was bathed in a gas of $\mathrm{H}$, He and $\mathrm{O}$. The dominant gas phase species were $\mathrm{H}_{2}, \mathrm{He}, \mathrm{H}_{2} \mathrm{O}$, and $\mathrm{CO}$. Thus grains in the accretion disk must have been exposed to and adsorbed $\mathrm{H}_{2}$ and water. We examine the efficacy of nebular gas adsorption as a mechanism by which the terrestrial planets accreted "wet". A simple model suggests that grains accreted to Earth could have adsorbed 1 - 3 Earth oceans of water. The fraction of this water retained during accretion is unknown, but these results suggest that at least some of the water in the terrestrial planets may have originated by adsorption.
\end{abstract}

Keywords. Earth, planets and satellites: formation

\section{Introduction}

Water is a common chemical compound in our solar system. In addition to Earth, it has been identified in asteroids, comets, meteorites, Mars, in the atmospheres, rings and moons of giant planets, and there is evidence for it in the poles of our Moon and of Mercury. The high deuterium to hydrogen $(\mathrm{D} / \mathrm{H})$ ratio of Venus atmosphere has been interpreted as evidence for Venus once having had far more water in the past than is present today. Here we address the origin of water in the terrestrial planets.

There is no agreement on the origin of water in the terrestrial planets. Possible sources of water can be divided into endogenous and exogenous. Endogenous sources include direct adsorption of water from gas onto grains in the accretion disk and accretion of hydrous minerals forming in the inner solar system. Exogenous sources include comets, hydrous asteroids, and phyllosilicates migrating from the asteroid belt. Recent reviews discuss this topic (e.g., Drake 2005; Campins et al. 2004; Lunine et al. 2003; Drake \& Righter 2002; Pepin \& Porcelli 2002; Robert, Gautier \& Dubrulle 2000).

We know virtually nothing about water in Mercury and Venus. We do have information on Martian water through studies of the Martian meteorites. However, Mars presents unique challenges because compositional information is more limited than for Earth. An important example is the global value of the $\mathrm{D} / \mathrm{H}$ ratio for Martian water. It is possible that we do not know Mars' intrinsic D/H isotopic ratio. Unlike Earth, Mars lacks plate tectonics and, hence, has no means of cycling water between mantle and crust. The Martian meteorites we measure on Earth may simply be sampling water delivered to Mars by cometary and asteroidal impacts subsequent to planetary formation. Note, however, that the contrary has been argued (Watson et al. 1994), i.e., that Martian 


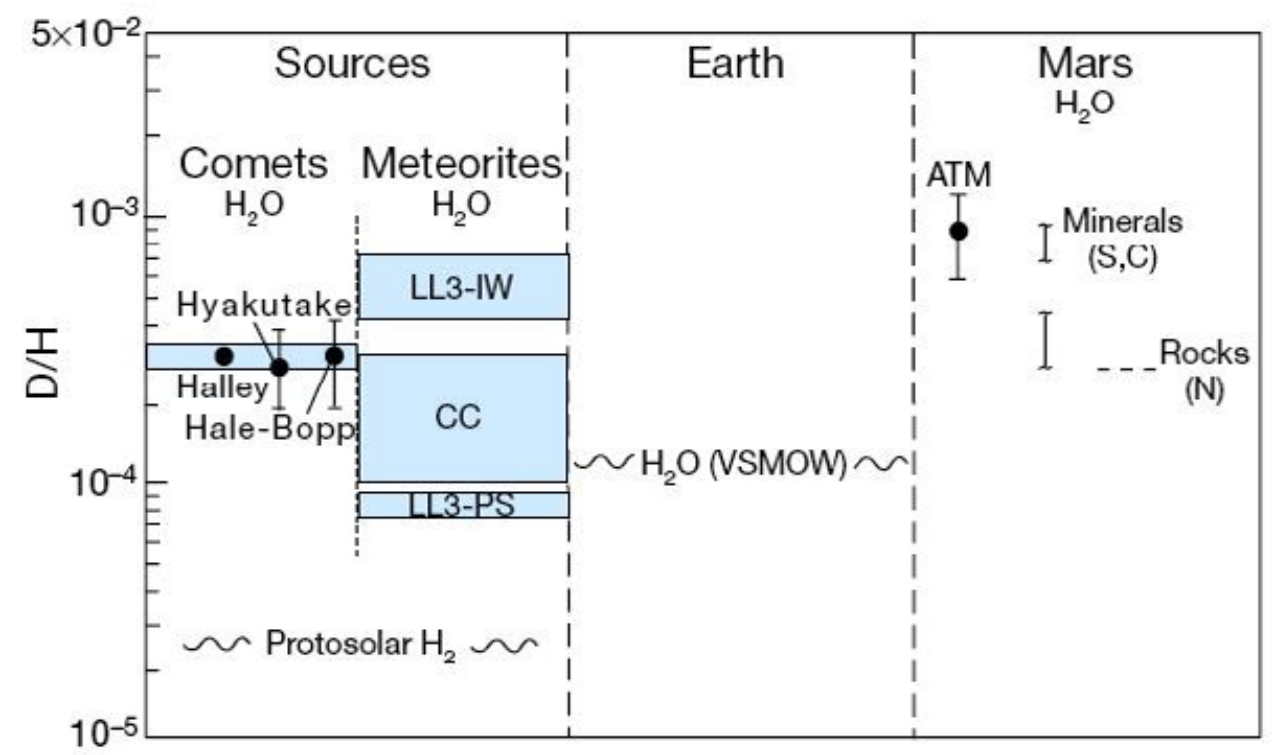

Figure 1. The D/H ratios in $\mathrm{H}_{2} \mathrm{O}$ in three comets, meteorites, Earth (Vienna standard mean ocean water - VSMOW), protosolar $\mathrm{H}_{2}$, and Mars. "CC" = carbonaceous chondrites, "LL3-IW" = interstellar water in Semarkona, "LL3-PS" = protostellar water in Semarkona. After Drake \& Righter (2002).

meteorites provide evidence for an intrinsic Martian mantle D/H ratio up to $50 \%$ higher than Earth's mantle (see Figure 1).

It seems likely that the origin of water on the other terrestrial planets cannot be divorced from the origin of water on Earth. Because we know most about water on Earth, the rest of this paper will be spent examining the origin of such water.

\section{Water on Earth}

\subsection{Accretion History}

The accretion of the Earth was dominated by a violent series of events. Dynamical theory of planetary accretion points inexorably to a hierarchy of accreting planetesimals, with the largest object accreting at any given time during the growth of a planet being one tenth to one third of the mass of the growing planet (Wetherill 1985; Chambers 2001). During the later stages of accretion, these collisions deposited enough energy to at least partly melt the Earth and, possibly, to have completely melted the Earth. The Earth probably experienced serial magma ocean events. The final, most massive impact ejected material into Earth orbit. This disk of material subsequently accreted to form the Moon (Canup \& Asphaug 2001).

Metal delivered by accreting planetesimals sank through these serial magma oceans and ponded at their bases for some period of time before transiting diapirically through the lower mantle to the center of the planet. Metal appears to have equilibrated with silicate at the base of the magma ocean. The mean depth calculated corresponds to at least the depth of the current upper mantle/lower mantle boundary of the Earth (Drake 
Table 1. Ratios of ${ }^{129} \mathrm{Xe}$ to ${ }^{132} \mathrm{Xe}$ in Earth and Mars reservoirs. OIB refers to ocean island basalts. MORB refers to mid-ocean ridge basalts After Musselwhite et al. (1991).

\begin{tabular}{lll}
\hline & Mars & Earth \\
\hline Atmosphere & 2.4 & 0.985 \\
& & \\
Basalts & $1.0-1.5$ & 0.988 OIB $^{*}$ \\
& & $1.00-1.14$ MORB $^{*}$ \\
\hline
\end{tabular}

2000). That depth probably represents some ensemble average memory of metal-silicate equilibrium in a series of magma oceans and should not be taken as the literal depth of the last magma ocean. Comparable conclusions can be drawn for the Earth's Moon, Mars, and Vesta (Righter \& Drake 1996).

As discussed below, the primitive atmosphere and ocean appear to have formed very early in Earth's history. Core formation, magma ocean solidification, ocean and atmospheric outgassing were essentially complete by $4.45 \mathrm{Ga}$ ago.

\subsection{Evidence for an Early Water Ocean}

It had long been thought that the accretion disk at $1 \mathrm{AU}$ was too hot for hydrous phases to be stable (e.g., Boss 1998), and that water was delivered to Earth after it formed by bombardment from asteroids and/or comets. However, recent geochemical evidence increasingly argues against asteroids and comets being the main sources of Earths water and points to the Earth accreting "wet" throughout its growth (Drake \& Righter 2002; Drake 2005). In either case the Earth may have had water oceans very early in its history.

Evidence for the existence of an early water ocean on Earth comes from the different reservoirs of ${ }^{129} \mathrm{Xe} /{ }^{132} \mathrm{Xe}$ in the atmosphere, mid-ocean ridge basalt (MORB) source, and ocean island basalt (OIB) source (Table 1). ${ }^{129} \mathrm{I}$ has a half-life of about $16 \mathrm{Ma}$ and is produced only during nucleosynthesis in a precursor astrophysical environment. All ${ }^{129} \mathrm{I}$ would have decayed to ${ }^{129} \mathrm{Xe}$ within $5-7$ half-lives, or about $100 \mathrm{Ma}$ following nucleosynthesis. Thus distinct ${ }^{129} \mathrm{Xe} /{ }^{132} \mathrm{Xe}$ reservoirs on Earth (and Mars) must have formed within $100 \mathrm{Ma}$ of nucleosynthesis of ${ }^{129} \mathrm{I}$.

It is difficult, although not impossible, to fractionate I from Xe by purely magmatic processes (Musselwhite \& Drake 2000). The problem is that I and Xe are both volatile and incompatible (they both have low vaporization temperatures and both prefer magmas to solid mantle), although $\mathrm{Xe}$ is a little less incompatible. However, water is extremely effective at fractionating I from Xe (Musselwhite, Drake \& Swindle 1991) because I dissolves in liquid water (it is a halogen) while Xe bubbles through (it is a gas).

If accretion ceased while ${ }^{129} \mathrm{I}$ still existed and any magma ocean solidified, liquid water could become stable at the Earths surface. Musslewhite (1995) showed that outgassed ${ }^{129}$ I could be recycled hydrothermally into the oceanic crust and subducted back into the mantle, which was by now partially degassed of Xe. Subsequent decay of ${ }^{129}$ I would give a MORB source with an elevated ${ }^{129} \mathrm{Xe} /{ }^{132} \mathrm{Xe}$ ratio in the mantle relative to the earlier outgassed atmosphere (Fig 2). A related conclusion was drawn for Mars (Musselwhite, Drake \& Swindle 1991). It appears that the Earth had a primitive atmosphere and large bodies of water by $4.45 \mathrm{Ga}$ ago.

Further evidence for the existence of an early water ocean on Earth comes from detrital zircons. Wilde et al. (2001) and Mojzsis, Harrison \& Pidgeon (2001) independently reported zircons of $4.4 \mathrm{Ga}$ age and $4.3 \mathrm{Ga}$ age respectively. On the basis of magmatic 


\section{lodine Recycling}

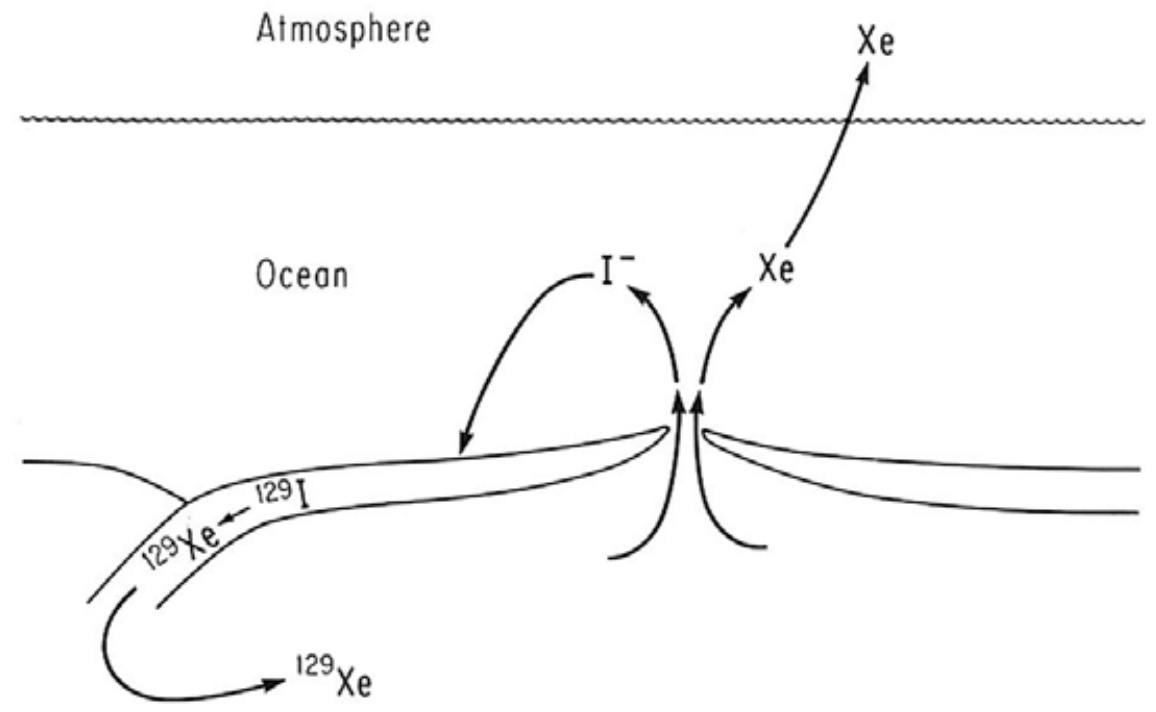

Figure 2. Cartoon illustrating outgassing of I and Xe while ${ }^{129} \mathrm{I}$ was still available. Xenon bubbles through the water ocean into the atmosphere. ${ }^{129} \mathrm{I}$ dissolves in water and is recycled hydrothermally into the crust and subducted back into the mantle. Subsequent decay of ${ }^{129} \mathrm{I}$ leads to mid-ocean ridge basalts (MORB) being erupted with elevated ${ }^{129} \mathrm{Xe} /{ }^{132} \mathrm{Xe}$ compared to the atmosphere. Separate Xe reservoirs imply fractionation of I from Xe within $100 \mathrm{Ma}$ after nucleosynthesis of ${ }^{129}$ I. From Musselwhite (1995).

oxygen isotope ratios and micro-inclusions of $\mathrm{SiO}_{2}$, these authors concluded that the zircons formed from magmas involving re-worked continental crust that had undergone low temperature interaction with a liquid hydrosphere. Wilde et al. (2001) specifically conclude that the $4.4 \mathrm{Ga}$ zircons represent the earliest evidence of both continental crust and water oceans on Earth.

Support for this conclusion was provided by Watson \& Harrison (2005), who used a geothermometer based on the Ti-content of zircons to conclude that the zircons crystallized at $\sim 700^{\circ} \mathrm{C}$, a temperature indistinguishable from granitoid zircon growth today. In other words, water-bearing evolved magmas were present within 200 Ma of solar system formation. The implication is that modern patterns of crust formation, erosion, and sediment formation had already been established prior to $4.4 \mathrm{Ga}$, implying liquid water oceans already existed.

It is not possible to estimate the amount of water outgassed by $4.4 \mathrm{Ga}$ ago. Water has been outgassed from the terrestrial mantle over the age of the solar system, but it has also been recycled back into the interior through subduction of oceanic plates at plate margins. Because the downgoing slab gets outgassed during subduction, not all water is transferred back to the mantle. The storage potential for water of silicates located primarily in the transition zone between lower and upper mantle is about 5 Earth oceans (Ohtani 2005). The time-dependent fluxes of water from the interior of the Earth to the surface and back cannot at present be deciphered. 


\section{Proposed Sources of Water and Methods of Discrimination}

\subsection{Discriminators}

In principle, it should be possible to determine the main sources of and relative contributions to Earth's water if they have distinct chemical and isotopic signatures. Signatures that are used as discriminators include:

a) the $\mathrm{D} / \mathrm{H}$ ratio of water in Earth, Mars, comets, meteorites, and the solar nebula

b) the relative abundances and isotopic ratios for noble gases on Earth, Mars meteorites, comets and the solar nebula

c) the ratio of noble gases to water on Earth, meteorites and comets

d) the isotopic composition of the highly siderophile (very strongly metal-seeking) element Os in Earth's primitive upper mantle (PUM), in the Martian mantle, and in meteorites

\subsubsection{D/H Ratios}

All deuterium is believed to have formed in the early universe. Stellar nuclear reactions convert $\mathrm{D}$ into $\mathrm{He}$, thus lowering the $\mathrm{D} / \mathrm{H}$ ratio of the universe with time. However, fractionation processes can produce local enhancements in the $\mathrm{D} / \mathrm{H}$ ratio. For example, low-temperature ion-molecule reactions in the cores of molecular clouds can enhance the $\mathrm{D} / \mathrm{H}$ ratio in icy grains by as much as two orders of magnitude above that observed in the interstellar medium (e.g., Gensheimer, Mauersberger \& Wilson 1996). In our solar system, there is evidence for more than one reservoir of hydrogen (Drouart, Dubrulle, Gautier, et al. 1999; Mousis et al. 2000; Robert 2001; Hersant, Gautier \& Hure 2001 and references therein). The solar nebula gas $\mathrm{D} / \mathrm{H}$ ratio is estimated from observations of $\mathrm{CH}_{4}$ in Jupiter and Saturn to be a low $2.1 \pm 0.4 \times 10^{-5}$ (Lellouch et al. 2001). Jupiter and Saturn likely obtained most of their hydrogen directly from solar nebula gas (this estimate is also consistent with protosolar $\mathrm{D} / \mathrm{H}$ value inferred from the solar wind implanted into lunar soils; Geiss \& Gloecker 1998). A second reservoir, enriched in D compared with the solar nebula gas, contributed to bodies that accreted from solid grains, including comets and meteorites.

We have $\mathrm{D} / \mathrm{H}$ ratios from water in three comets (all from the Oort cloud), Halley $\left(3.2 \pm 0.1 \times 10^{-4}\right.$, Eberhardt et al. 1995); Hyakutake $\left(2.9 \pm 1.0 \times 10^{-4}\right.$, Bockele-Morvan et al. 1998); and Hale-Bopp $\left(3.3 \pm 0.8 \times 10^{-4}\right.$, Meier et al. 1998). These are all about twice the value for terrestrial water $\left(1.49 \times 10^{-4}\right.$, Lecuyer, Gillet \& Robert 1998), about fifteen times the value for the solar nebula gas $\left(2.1 \pm 0.4 \times 10^{-5}\right.$, Lellouch et al. 2001), and consistent with the range of values for hot cores of dense molecular clouds $\left(2\right.$ to $6 \times 10^{-4}$, Gensheimer, Mauersberger \& Wilson 1996). Carbonaceous chondrites have the highest water abundance of all meteorites (up to $17 \mathrm{wt} \%$; Jarosewich 1990) and their D/H ratios range from $1.20 \times 10^{-4}$ to $3.2 \times 10^{-4}$ (Lecuyer, Gillet \& Robert 1998). The largest D enrichment in a water-bearing mineral in a meteorite was measured at $7.3 \pm 1.2 \times 10^{-4}$ in the LL3 chondrite Semarkona (Deloule \& Robert 1998). These results are illustrated in Figure 1.

The measurement of the $\mathrm{D} / \mathrm{H}$ ratio of water in three comets is a significant development. Different authors interpret these ratios in very different ways. Some (e.g., Dauphas, Robert \& Marty 2000; Morbidelli et al. 2000; Drake \& Righter 2002; Robert 2001) consider the high $\mathrm{D} / \mathrm{H}$ ratio in these comets as evidence against a cometary origin of most of the terrestrial water. Others (e.g., Delsemme 2000, Owen \& Bar-Nun 2001) argue that comets are the main reservoir of deuterium-rich water that raised the terrestrial $\mathrm{D} / \mathrm{H}$ a factor of six above the protosolar value. Complicating the matter further, recent laboratory measurements of the $\mathrm{D} / \mathrm{H}$ ratio in sublimating ices have shown that fractionation 


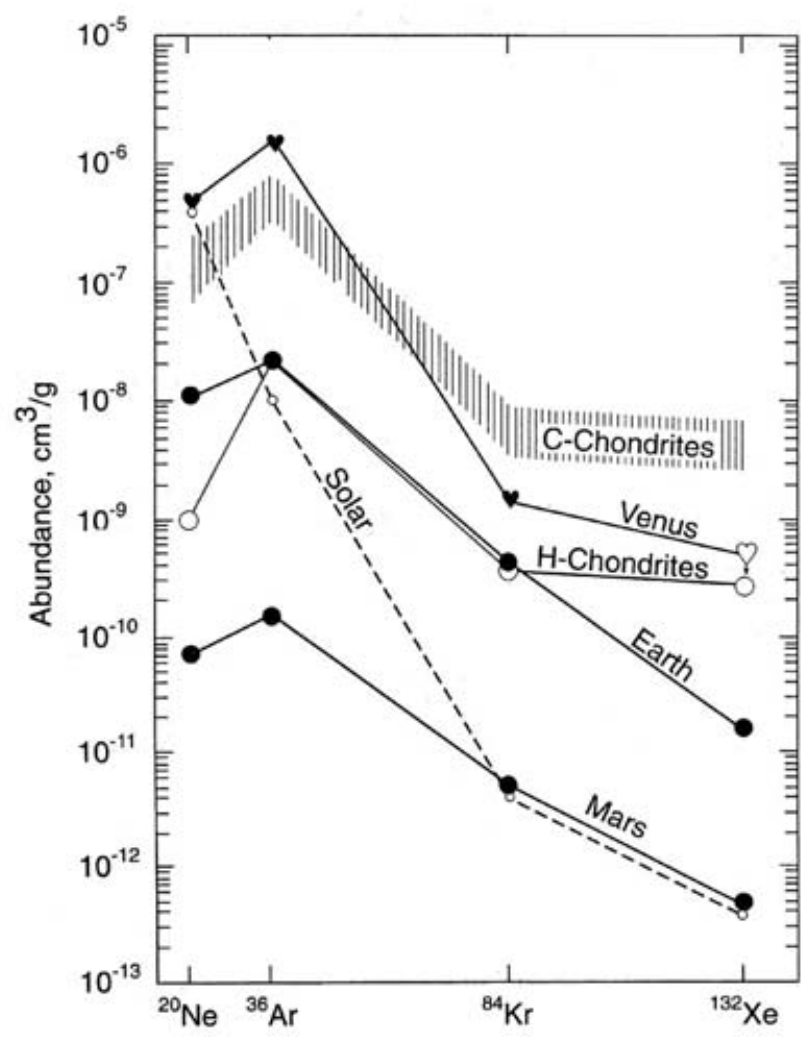

Figure 3. Noble gases in Venus, Earth, Mars, and meteorites. After Owen \& Bar-Nun (1995).

can occur during sublimation (Weirich, Brown \& Lauretta 2004; Moores, Brown, Lauretta, et al. 2005). During sublimation of water ice samples in a vacuum the $\mathrm{D} / \mathrm{H}$ ratio of the evolved gas varies with time and can increase or decrease relative to the initial $\mathrm{D} / \mathrm{H}$ ratio, depending on the nature of the sample. The root cause is interpreted to be differential diffusion and sublimation of $\mathrm{HDO}$ and $\mathrm{H}_{2} \mathrm{O}$. This result means that the measured $\mathrm{D} / \mathrm{H}$ ratios in cometary comae may not be representative of the bulk cometary values.

\subsubsection{Noble Gases}

Noble gases are chemically inert and very volatile. Hence they probably arrive at a planet along with other volatiles, quickly move to the planet's atmosphere, and avoid the chemical complications of planetary evolution. Thus the noble gas characteristics of a planetary atmosphere can be tracers of the source of the planet's volatiles.

Figure 3 shows the abundances of noble gases in Venus, Earth and Mars compared with solar abundances and those in two kinds of meteorites. Note that the proportions of $\mathrm{Ar}, \mathrm{Kr}$, and Xe in the atmospheres of Earth and Mars are remarkably similar, and completely different from the abundances found in meteorites or the Sun (solar wind). We concentrate here on the possibility of cometary and meteoritic contributions to Earth and Mars.

We do not know much about noble gases in comets, and some of the measurements we do have appear contradictory. Krasnopolsky et al. (1997) reported an upper limit in comet Hale-Bopp of $0.5 \%$ of the solar Ne/O ratio. Stern et al. (2000) reported a tentative 
Ar detection in comet Hale-Bopp and a roughly solar Ar/O ratio. Weaver et al. (2002) reported upper limits for $\mathrm{Ar} / \mathrm{O}$ of $<10 \%$ and $<8 \%$ of the solar value in comets LINEAR 2001 A2 and LINEAR 2000 WM1, respectively. All these observations have been made of comets from the Oort cloud. The more sensitive upper limits for Ar in the LINEAR comets are not consistent with the detection reported by Stern et al. (2000) in comet Hale-Bopp. At this point it is not clear if comet Hale-Bopp was unusually rich in Ar or if the tentative detection is somehow flawed.

\subsubsection{Siderophile Elements in Earths Mantle}

The relatively high abundances of highly siderophile (very strongly metal-seeking) elements (HSEs) at $0.003 \times$ CI in Earth's primitive upper mantle (PUM) and their roughly chondritic element ratios suggest that these elements arrived after Earth's core formation had ceased (e.g., Drake \& Righter 2002). Had these elements arrived sooner they would have been quantitatively extracted into Earth's core. This material is commonly termed the "late veneer". Drake \& Righter (2002) argue that Earth-building materials shared some but not all the properties with extant meteorites, i.e., no primitive material similar to Earth's mantle is currently in our meteorite collections. More specifically, Re and Os are two HSEs that are linked by beta decay, ${ }^{187} \mathrm{Re}={ }^{187} \mathrm{Os}+\beta$ thus Os isotopes can be used to constrain the origin of the "late veneer". Carbonaceous chondrites, the only abundant water-bearing meteorites, have a significantly lower ${ }^{187} \mathrm{Os} /{ }^{188} \mathrm{Os}$ ratio of 0.1265 than Earth's primitive upper mantle (PUM) value of 0.1295 , effectively ruling them out as the source of the "late veneer" (Fig. 4). [Note that external \pm 2 sigma precision on the ${ }^{187} \mathrm{Os} /{ }^{188} \mathrm{Os}$ ratio is within the size of each individual data point in the histogram (Brandon et al. 2005).] Mars primitive upper mantle appears to have an even higher ${ }^{187} \mathrm{Os} /{ }^{188} \mathrm{Os}$ ratio of 0.132 (Brandon et al. 2005). The Earth's mantle ${ }^{187} \mathrm{Os} /{ }^{188} \mathrm{Os}$ ratio overlaps anhydrous ordinary chondrites and is distinctly higher than anhydrous enstatite chondrites, while the Martian mantle is higher than all common meteorite types.

\subsection{Sources}

In this section we examine the principal proposed sources of water in the terrestrial planets in the light of these discriminators. We divide these sources into two categories, endogenous and exogenous and we evaluate their possible contributions using the discriminators discussed in section 3.1 .

\subsubsection{Endogenous Sources}

\subsubsection{Primordial Gas Captured from the Solar Nebula}

It has been argued that a primordial atmosphere could not have been captured directly from the solar nebula, principally because of Earth's D/H ratio in water and noble gas abundances. However, Campins et al. (2004) point out that the processes involved in planetary accretion, degassing, and the evolution of a hydrosphere and atmosphere are complex and may have fractionated the chemical and isotopic signatures of the source(s) of water. Hydrogen, for example, may be an important constituent in the outer core and possibly inner core of Earth (e.g., Okuchi 1997, 1998). If H and D were fractionated in that process, the residual $\mathrm{D} / \mathrm{H}$ ratio in the hydrosphere may not reflect that of the original source. Following accretion, the surface of the Earth continued to be modified by large impacting asteroids and comets (Section 3.2.2). Large impact events have the capacity to completely volatilize any oceans (Zahnle \& Sleep 1997) and blow off portions of Earth's atmosphere (Melosh \& Vickery 1989). Fractionation of D/H and noble gas/water ratios may occur as a consequence of impact processes, which may also mask the signatures of the original source material. 


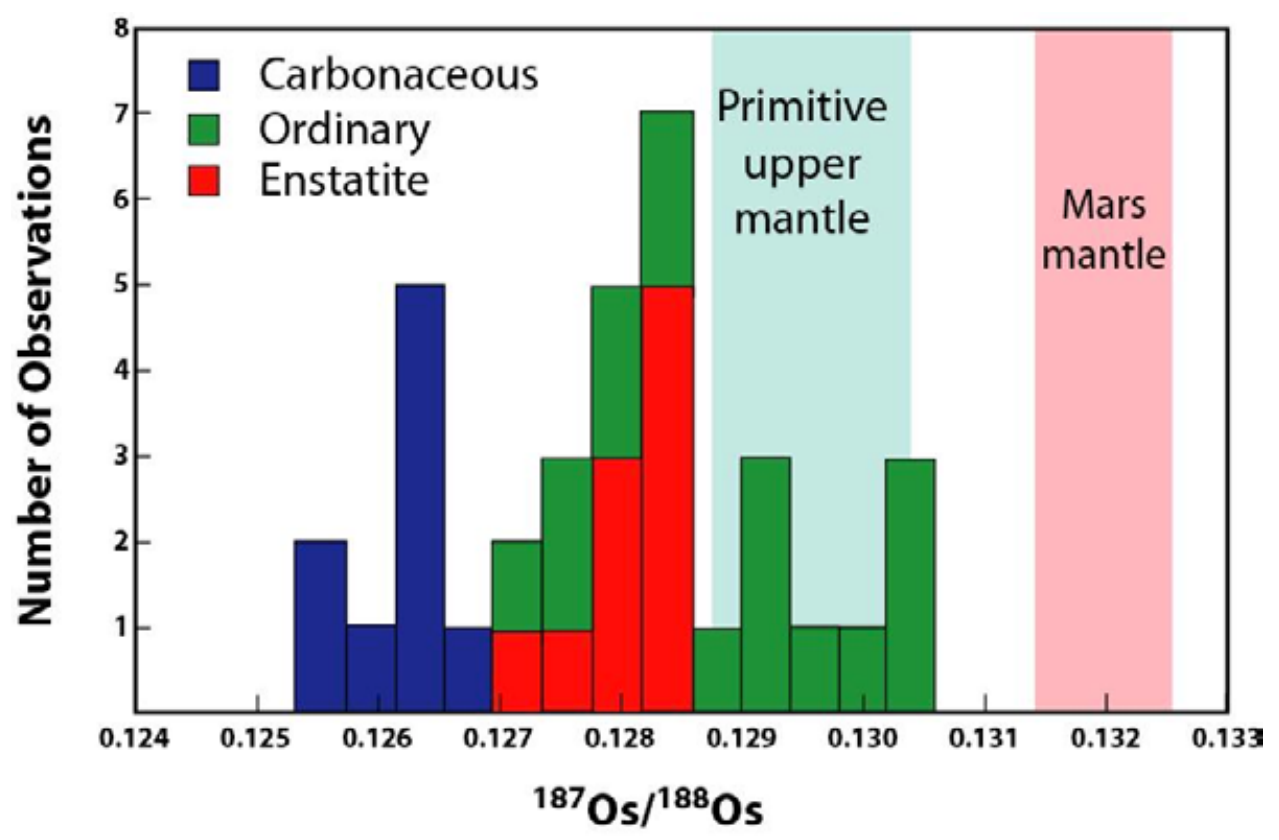

Figure 4. ${ }^{187} \mathrm{Os} /{ }^{188} \mathrm{Os}$ ratios in carbonaceous, ordinary, and enstatite chondrites, and in the Earth's primitive upper mantle (PUM), are distinct and are diagnostic of the nature of the Earth's "late veneer". Mars is not plotted because the uncertainty in its initial ${ }^{187} \mathrm{Os} /{ }^{188} \mathrm{Os}$ ratio is larger than range of the X-axis. After Drake and Righter (2002).

\subsubsection{Adsorption of Water onto Grains in the Accretion Disk}

The terrestrial planets grew in an accretion disk of gas and dust grains. Hydrogen, $\mathrm{He}$, and $\mathrm{O}_{2}$ dominated the gas in which the dust was bathed. Some of that $\mathrm{H}_{2}$ and $\mathrm{O}_{2}$ combined to make water vapor. If thermodynamic equilibrium was attained, there were about two Earth masses of water vapor in the accretion disk inside of 3 AU (Drake 2005). The mass of the Earth is $5 \times 10^{27} \mathrm{~g}$. The mass of one Earth ocean is $1.4 \times 10^{24} \mathrm{~g}$. The extreme maximum estimate for the amount of water in the Earth is about 50 Earth oceans (Abe et al. 2000), with most estimates being 10 Earth oceans or less. For example, an estimate based on the water storage potential of minerals in the silicate Earth is about 5 - 6 Earth oceans (Ohtani 2005). Thus the mass of water vapor available in the region of the terrestrial planets far exceeded the mass of water accreted.

Could water vapor be adsorbed onto grains before the gas in the inner solar system was dissipated? Stimpfl, Lauretta \& Drake (2004) have examined the role of physisorption by modeling the adsorption of water on to grains at $1000^{\circ} \mathrm{K}, 700^{\circ} \mathrm{K}$, and $500^{\circ} \mathrm{K}$ using a Monte Carlo simulation. Stimpfl, Lauretta \& Drake (2004) "exploded" the Earth into $0.1 \mu \mathrm{m}$ spheres of volume equal to Earth, recognizing that grains in the accretion disk are not spherical and would be fractal in nature. If the surface area of the fractal grain was 100 times that of a sphere of corresponding volume, then one quarter of an ocean of water could be adsorbed at $1000^{\circ} \mathrm{K}$, one Earth ocean could be adsorbed at $700^{\circ} \mathrm{K}$, and three Earth oceans could be adsorbed at $500^{\circ} \mathrm{K}$. Obviously in the accretion disk there would be a size-frequency distribution of grain sizes and variable fractal dimensions, but the exercise shows that the concept deserves further exploration. This work is discussed in more detail in Drake (2005).

Of course, there are issues of retention of water as the grains collide and grow to make planets. However, it is clear that volatiles are not completely outgassed even in 
planetary scale collisions. For example, primordial ${ }^{3} \mathrm{He}$, far more volatile than water, is still outgassing from Earth's mantle 4.5 Ga after an almost grown Earth collided with a Mars-sized body to make the Moon.

Stimpfl, Lauretta \& Drake (2004) showed that the efficiency of adsorption of water increases as temperature decreases, that is, the process should have been more efficient further from the Sun than closer to the Sun. Thus, it is likely that Mars, Earth, and Venus all accreted some water by adsorption, with Mars accreting the most both because of its greater distance from the Sun and the lower energy of collisions during accretion because of its smaller final mass. The current differences in the apparent water abundances among the terrestrial planets are probably the result of both different initial inventories and subsequent geologic and atmospheric processing.

There is an interesting consequence for the evolution of planetary redox states if the terrestrial planets accreted "wet". Okuchi (1997) showed that when Fe-metal and water were compressed to $30-100 \mathrm{kbars}$ and heated to $1200^{\circ} \mathrm{C}-1500^{\circ} \mathrm{C}$, iron hydride formed. In a magma ocean environment, metal sinking to form planetary cores should contain $\mathrm{H}$, and $\mathrm{OH}$ should be left behind in the molten silicate. As more metal was delivered to the planet as it accreted, more $\mathrm{H}$ would be extracted into the core and more $\mathrm{OH}$ liberated in the silicate. Thus planetary mantles should become progressively more oxidized with time, perhaps explaining the high redox states relative to the iron - wüstite buffer. This process might explain the correlation of the degree of oxidation of silicate mantles with planet mass (Righter, Drake \& Scott 2006).

\subsubsection{Exogenous Sources}

\subsubsection{Comets}

Comets were long considered the most likely source of water in the terrestrial planets. A cometary source was attractive because it is widely believed that the inner solar system was too hot for hydrous phases to be thermodynamically stable (Boss 1998). Thus an exogenous source of water was needed.

There are elemental and isotopic reasons why at best $50 \%$ and, most probably, a very small percentage of water accreted to Earth from cometary impacts (Drake \& Righter, 2002). Figure 1 compares the isotopic composition of hydrogen in Earth, Mars, three Oort Cloud comets, and various early solar system estimates. It is clear that $100 \%$ of Earth's water did not come from Oort Cloud comets with $\mathrm{D} / \mathrm{H}$ ratios like the three comets measured so far. $\mathrm{D} / \mathrm{H}$ ratios in Martian meteorites do agree with the three cometary values (Figure 1). That may reflect the impact of comets onto the Martian surface in a non-plate tectonics environment that precludes recycling of surface material into the Martian mantle (Drake 2005). Conversely, there are models (Lunine et al. 2003) that have asteroids and comets from beyond 2.5 AU as the main source of Mars' water.

So what limits the cometary contribution to Earth's water? Consider, for example, that perhaps Earth accreted some hydrous phases or adsorbed water, and some amount of additional water came from comets. Indigenous Earth water could have had D/H ratios representative of the inner solar system, i.e., low values because of relatively high nebular temperatures, perhaps like protosolar hydrogen $\left(2-3 \times 10^{-5}\right.$, Lecluse \& Robert 1994) in which case a cometary contribution of up to $50 \%$ is possible. Alternatively, indigenous Earth water could have had $\mathrm{D} / \mathrm{H}$ ratios representative of a protosolar water component identified in meteorites $\left(\sim 9 \times 10^{-5}\right.$, Deloule \& Robert 1995), in which case there could be as little as a $10-15 \%$ cometary contribution (Owen \& Bar-Nun 2000).

There are caveats to using cometary $\mathrm{D} / \mathrm{H}$ ratios to limit the delivery of cometary water to Earth. First, we do not know that Oort Cloud comets Halley, Hale-Bopp, and Hyakutake are representative of all comets. Certainly they are unlikely to be representative of 
Kuiper Belt objects, the source of Jupiter family comets, as Oort Cloud comets formed in the region of the giant planets and were ejected while Kuiper Belt objects have always resided beyond the orbit of Neptune. Second, D/H measurements are not made of the solid nucleus, but of gases emitted during sublimation. As mentioned in Section 3.1, differential diffusion and sublimation of $\mathrm{HDO}$ and $\mathrm{H}_{2} \mathrm{O}$ may make such measurements unrepresentative of the bulk comet. The $\mathrm{D} / \mathrm{H}$ ratio would be expected to rise in diffusion and sublimation, as has been confirmed in preliminary laboratory experiments on pure water ice (Weirich, Brown \& Lauretta 2004). Lower bulk D/H ratios would increase the allowable amount of cometary water. Intriguing experiments on mixtures of water ice and $\mathrm{TiO}_{2}$ grains by Moores et al. (2005) suggest that $\mathrm{D} / \mathrm{H}$ ratios could be lowered in sublimates. Third, the $\mathrm{D} / \mathrm{H}$ ratios of organics and hydrated silicates in comets are unknown, although that situation may be rectified by analysis of samples returned by the Stardust mission. Note, however, that $\mathrm{D} / \mathrm{H}$ ratios up to $50 \times$ Vienna Standard Mean Ocean Water (VSMOW) have been measured in some chondritic porous interplanetary dust particles (CP-IDPs) which may have cometary origins (Messenger 2000), and higher aggregate $\mathrm{D} / \mathrm{H}$ ratios of comets would decrease the allowable cometary contribution to Earth's water.

Delivery of water from comets can also be evaluated in light of other cometary geochemical data. For an assumed $\mathrm{Ar} / \mathrm{H}_{2} \mathrm{O}$ ratio of $1.2 \times 10^{-7}$ in the bulk Earth, comets like Hale-Bopp with an approximately solar ratio of $\mathrm{Ar} / \mathrm{H}_{2} \mathrm{O}$ (Stern, Slanter, Festou, et al. 2000 ) would bring in $2 \times 10^{4}$ more Ar than is presently in the Earths atmosphere (Swindle \& Kring 2001), if 50\% of Earth's water, the maximum amount permitted by D/H ratios, was derived from comets. It is unclear if this measurement of comet Hale-Bopp is applicable to all comets. However, the true Ar/O ratios in comets would have to be at least three orders of magnitude below solar in order to be consistent with the Ar abundance of the Earth's atmosphere.

Another approach to estimating the contribution of cometary materials to Earth's water budget can be made by considering the implications for the abundances of noble metals and noble gases. Dauphas \& Marty (2002) show that the total mass of cometary and asteroidal material accreting to Earth after core formation is $0.7-2.7 \times 10^{25} \mathrm{~g}$ and that comets contribute $<0.001$ by mass or $<0.7-2.7 \times 10^{22} \mathrm{~g}$. Given that the minimum mass of water in the Earth, one Earth ocean, is $1.4 \times 10^{24} \mathrm{~g}$, comets can contribute less than $1 \%$ of Earth's water.

\subsubsection{Asteroids}

Asteroids are a plausible source of water based on dynamical arguments. Morbidelli et al. (2000) have shown that up to $15 \%$ of the mass of the Earth could be accreted late in Earths growth by collision of one or a few asteroids originating in the Main Belt. However, there are strong geochemical arguments against a significant contribution of water from asteroids, unless one postulates that Earth was hit by a hydrous asteroid unlike any falling to Earth today as sampled by meteorites. One cannot prove this hypothesis wrong, as it could involve a single, unique event.

However, if asteroidal material falling to Earth 4.5 Ga ago was the same as that which falls today, one can effectively rule out asteroids as a source of water. The reason involves the Os isotopic composition of the so-called "late veneer", the material that may have contributed the highly siderophile elements (HSEs) that are present to within $4 \%$ of chondritic proportions at about 0.003 of chondritic absolute abundances (Fig. 5).

Of current meteorite falls, only carbonaceous chondrites have significant amounts of water (Jarosewich 1990). Enstatite and ordinary chondrites are essentially anhydrous. The late addition of water must be accompanied by other chemical elements such as Re 


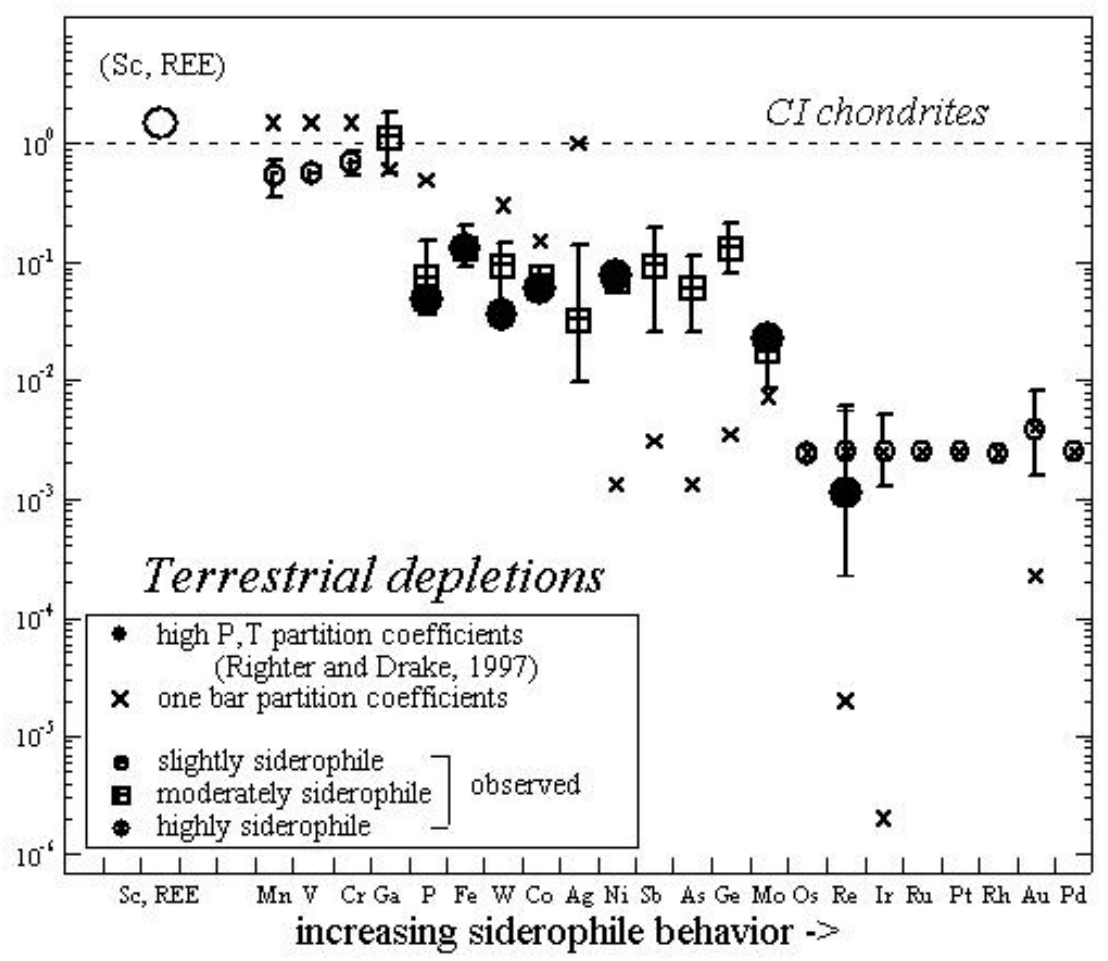

Figure 5. Comparison of the observed siderophile element depletions in Earth's upper mantle (open symbols - abundances normalized to CI chondrites and refractory elements), with those calculated using one bar partition coefficients (crosses) and high pressure / high temperature calculated partition coefficients (solid circles). Calculated depletions using the latter partition coefficients overlap the observed depletions, consistent with metal-silicate equilibrium and homogeneous accretion. After Drake (2000).

and Os. Earth's Primitive Upper Mantle (PUM) has a significantly higher ${ }^{187} \mathrm{Os} /{ }^{188} \mathrm{Os}$ ratio than carbonaceous chondrites, effectively ruling them out as the source of the "late veneer" (Fig. 4). The PUM ${ }^{187} \mathrm{Os} /{ }^{188}$ Os ratio overlaps anhydrous ordinary chondrites and is distinctly higher than anhydrous enstatite chondrites. Mars' PUM has an even higher ${ }^{187} \mathrm{Os} /{ }^{188}$ Os ratio, making an asteroidal source of Martian water even more unlikely.

The identification of anhydrous meteorites with the "late veneer" (Walker et al. 2002) effectively rules out asteroidal material being a late source of Earth's and Mars' water. There is one caveat, however; thermal processing of asteroids was occurring $4.5 \mathrm{Ga}$ ago. One cannot exclude the possibility that ordinary chondrites once contained water and those falling to Earth today have lost it by metamorphism, even though it was still present $4.5 \mathrm{Ga}$ ago. However, the preservation of aqueous alteration products in some carbonaceous chondrites (McSween 1979) suggests that loss of water from initially hydrous asteroids is unlikely to proceed to the anhydrous limit.

\subsubsection{Early Accretion of Water from Inward Migration of Hydrated Phyllosilicates}

Most solar nebula models suggested that the growth zones of the terrestrial planets were too hot for hydrous minerals to form (e.g., Cyr, Sears \& Lunine 1998; Delsemme 2000; Cuzzi \& Zahnle 2004). Ciesla \& Lauretta (2004) suggest that hydrous minerals were formed in the outer asteroid belt region of the solar nebula and were then transported to the hotter regions of the nebula (i.e., Earth and Mars) by gas drag, where they were incorporated into the planetesimals that formed there. These hydrated minerals were 
able to survive for long periods in hotter regions due to sluggish dehydration kinetics. Note that this mechanism differs from the delivery of water by stochastic impacts with large planetary embryos originating in the outer asteroid belt region (Section 3.2.2.2). Drake (2005) points out that it seems unlikely that phyllosilicates could be decoupled from other minerals and transported into the inner solar system. Thus the proposed radial migration of hydrated minerals appears subject to the same objection involving Os isotopes discussed in Section 3.2.2.2.

\subsubsection{Water and Organics}

It has been postulated that water and organics were delivered from the same cometary and/or asteroidal source (e.g., Chyba 1993; Delsemme 2000). In light of the new evidence, such combined delivery seems less likely. In addition to comets and asteroids being inconsistent with some geochemical properties of the Earth, it is unlikely that a complex organic material would have survived the magma ocean accompanying the formation of the Moon at the end of accretion of the Earth (Drake 2000). However, it seems possible that complex organic material may have been delivered to Earth after it formed and liquid water oceans became stable.

Comets, which are known to be rich in organic molecules, have been postulated to be the principal source of terrestrial amino acids (Pierazzo \& Chyba 1999; Chyba 1993). Some meteorites are also rich in carbon and organic compounds, hence, asteroids may have also contributed significantly to Earths organic inventory. In fact, Kring \& Cohen (2002) point out that during late heavy bombardment, asteroidal material probably delivered a large mass of organic material to Earths surface, as much as 160 times larger than that in the total land biomass today. Even if some of the organic molecules were dissociated during the impacts, Kring \& Cohen (2002) propose the formation of impactgenerated hydrothermal regions with lifetimes up to $10^{6}$ years, where complex organic molecules might reassemble.

\subsection{Outstanding Challenges}

Geochemical data for the Earth, Mars, meteorites, and comets cast increasing doubt on either asteroids or comets being the principal source of water in the terrestrial planets. The idea that the terrestrial planets could have obtained much or all of their water by adsorption on to grains directly from gas in the accretion disk is relatively new (Stimpfl, Lauretta \& Drake 2004; Drake 2005). Many major issues remain to be resolved in order to make further progress. Below we list some of the most important.

1. The $\mathrm{D} / \mathrm{H}$ ratio of the nebular gas is inferred from spectroscopic measurements of $\mathrm{CH}_{4}$ in the atmospheres of Jupiter and Saturn to be $2.1 \pm 0.4 \times 10^{-5}$ (Lellouch et al. 2001), much lower than VSMOW. Spectroscopic or direct measurements of solar D/H cannot be made because practically all of the Suns deuterium has been burned to make He. If the nebular D/H ratio really is as low as implied by the Jovian and Saturnian atmospheres, a mechanism to raise the $\mathrm{D} / \mathrm{H}$ ratio of nebular gas from solar to VSMOW is needed.

2. It is likely that the $\mathrm{D} / \mathrm{H}$ and $\mathrm{Ar} / \mathrm{O}$ ratios measured in cometary comas and tails are not truly representative of cometary interiors. Reconnaissance experiments have been shown that $\mathrm{D} / \mathrm{H}$ ratios in laboratory experiments can increase or decrease with time due to differential diffusion and sublimation, depending on the physical nature of the starting material (Weirich, Brown \& Lauretta 2004; Moores et al. 2005). Further, measurements of $\mathrm{Ar} / \mathrm{O}$ ratios in comets are either upper limits or 3 sigma detection limits and are somewhat uncertain. Depending on the siting of Ar in comets, Ar/O ratios may also be unrepresentative of cometary interiors. The Deep Impact mission will be the first attempt to expose fresh cometary interior material for spectral analysis with ground-based and 
space-based high spectral resolution spectrometers. It is unclear if improvement in our understanding of cometary $\mathrm{D} / \mathrm{H}$ ratios and $\mathrm{Ar} / \mathrm{O}$ ratios will be made.

3. The key argument against an asteroidal source of Earths water is that the Os isotopic composition of Earths primitive upper mantle matches that of anhydrous ordinary chondrites, not hydrous carbonaceous chondrites. But are the parent bodies of the ordinary chondrites anhydrous? Could ordinary chondritic meteorites be derived from the metamorphosed outer parts of hydrous asteroids, in which case impact of a bulk asteroid could deliver water? It is probable that spacecraft spectral examination of very deep impact basins in S-type asteroids will be needed to address this question.

4. A related question is why there are any anhydrous primordial bodies, such as the parent bodies of anhydrous meteorites, in the solar system if adsorption of water from gas in the accretion disk was an efficient process, as preliminary calculations suggest it might have been.

5. The timing of loss of gas from the accretion disk in the region of the terrestrial planets is unknown. For adsorption to be efficient, nebular gas must persist long enough for grains to adsorb water. Radial migration of phyllosilicates also depends on the presence of gas. The timing of loss of gas from the accretion disk will be intimately connected to the currently unknown mechanism of loss.

\section{References}

Abe, Y., Ohtani, E., Okuchi, T., Righter, K., \& Drake, M.J. 2000, in: R.M. Canup \& R. Righter (eds.), Origin of the Earth and Moon (Tucson: Univ. Arizona Press), p. 413

Bockelee-Morvan, D., Gautier, D., Lis, D.C., Young, K., Keene, J., Phillips, T., Owen, T., Crovisier, J., Goldsmith, P.F., Bergin, E.A., Despois, D., \& Wooten, A. 1998, Icarus 133, 147

Boss, A.P. 1998, Ann. Rev. Earth Planet. Sci. 26, 53

Brandon, A.D., Humayun, M., Puchtel, I.S., \& Zolensky, M.E. 2005, Geochim. Cosmochim. Acta 69,1619

Campins, H., Swindle, T.D., \& Kring, D.A. 2004, in: J. Seckbach (ed.) Origin, Evolution and Biodiversity of Microbial Life in the Universe, p. 569

Canup, R.M. \& Asphaug, E. 2001, Nature 412, 708

Chambers, J.E. 2001, Icarus 152, 205

Chyba 1993, Geochimica et Cosmochimica Acta 57, 3351

Ciesla, F.J., Lauretta D.S., \& Hood L.L. 2004, Lunar Planet. Sci. XXXV, abstract 1219.

Cuzzi, J.N. \& Zahnle, K.J. 2004, Ap. J. 614, 490

Cyr, K.E., Sears, W.D., \& Lunine, J.I. 1998, Icarus 135, 537

Dauphas, N. \& Marty B. 2002, Jour. Geophys. Res. 107, E12-1

Dauphas, N., Robert, F., \& Marty, B. 2000, Icarus 148, 508

Deloule, E. \& Robert F. 1995, Geochim. Cosmochim. Acta 59, 4695

Delsemme, A.H. 2000, Icarus 146, 313

Drake, M.J. 2000, Geochim. Cosmochim. Acta 64, 2363

Drake, M.J. 2005, Meteoritics and Planetary Science 40, 519

Drake, M.J. \& Righter, K. 2002, Nature 416, 39

Drouart, A., Dubrulle, B., Gautier, D., \& Robert, F. 1999, Icarus 140, 129

Eberhardt, P., Reber, M., Krankowsky, D., \& Hodges, R.R. 1995, Astron. EJ Astrophys. 302, 301

Geiss, J. \& Gloeckler, G. 1998, Space Science Reviews 84, 239

Gensheimer, P.D., Mauersberger, R., \& Wilson, T.L. 1996, Astron. $\mathcal{E}$ Astrophys. 314, 281

Hersant, F., Gautier, D., \& Huré, J.-M. 2001, Ap.J. 554, 391

Jarosewich, E. 1990, Meteoritics 25, 323

Krasnopolsky, V.A., Mumma, M.J., Abbott, M., Flynn, B.C., Meech, K.J., Yeomans, D.K., Feldman, P.D., \& Cosmovici, C.B. 1997, Science 277, 1488

Kring, D.A. \& Cohen B.A. 2002, J. Geophys. Res. 107, 4-1 
Lecluse, C. \& Robert, F. 1994, Geochim. Cosmochim. Acta 58, 2927

Lecuyer, C., Gillet, P., \& Robert, F. 1998, Chem. Geol. 145, 249

Lellouch, E., Bézard B., Fouchet T., Feuchtgruber H., Encrenaz T., \& de Graauw T. 2001, Astron. ES Astrophys. 370, 610

Lunine, J.I., Chambers, J., Morbidelli, A., \& Leshin, L.A. 2003, Icarus 165, 1

McSween, H.Y. 1979, Revs. Geophys. Space Phys. 17, 1059

Meier, R., Owen, T.C., Matthews, H.E., Jewitt, D.C., Bockelee-Morvan, D., Biver, N., Crovisier, \& Gautier, D. 1998, Science 279, 842

Melosh, H.J. \& Vickery, A.M. 1989, Nature 338, 487

Messenger, S. 2000, Nature 404, 968

Mojzsis, S.J., Harrison, T.M., \& Pidgeon, R.T. 2001, Nature 409, 178.

Moores, J.E., Brown, R.H., Lauretta, D.S., \& Smith, P.H. 2005, Lunar and Planetary Science $X X X V I$, abstract 1973

Morbidelli, A., Chambers J., Lunine J.I., Petit J.M., Robert F., Valsecchi G.B., \& Cyr K.E. 2000, Meteoritics and Planetary Science 35, 1309

Mousis, O., Gautier, D., Bockelée-Morvan, D., Robert, F., Dubrulle, B., \& Drouart, A. 2000, Icarus 148,513

Musselwhite, D.S. \& Drake, M.J. 2000, Icarus 148, 160

Musselwhite, D.S., Drake, M.J., \& Swindle, T.D. 1991, Nature 352, 697

Musselwhite, D.S. 1995, Ph.D. thesis, University of Arizona, Tucson.

Ohtani, E. 2005, Elements 1, 25

Okuchi, T. 1997, Science 278, 1781

Okuchi, T. 1998, J. Phys. Condensed Matter 10, 11595

Owen, T. \& Bar-Nun, A. 2001, Origins Life Evol. Biosphere 31, 435

Owen, T. \& Bar-Nun, A. 2000, in: R.M. Canup \& K. Righter (eds.) Origin of the Earth and Moon (Tucson: Univ. of Arizona Press), p. 459

Owen, T. \& Bar-Nun, A. 1995, Icarus 116, 215

Pepin, R.O. \& Porcelli D. 2002, Reviews in Mineralogy and Geochemistry 47, 191

Pierazzo \& Chyba 1999, Meteoritics and Planetary Science 34, 909

Righter, K. \& Drake, M.J. 1996, Icarus 124, 513

Righter, K., Drake, M.J., \& Scott E. 2006, in: L. Leshin \& D. Lauretta (eds.), Meteorites and the Earth Solar System II (Tucson: Univ. of Arizona Press), (submitted).

Robert, F. 2001, Science 293, 105

Robert, F., Gautier, D., \& Dubrulle, B. 2000, Space Sci. Rev. 92, 201

Stern, S.A., Slater D.C., Festou M.C., Parker J.W., Gladstone G.R., A'Hearn M.F., \& Wilkinson E. $2000, A p J .544$, L169

Stimpfl, M., Lauretta, D.S., \& Drake, M.J. 2004, Meteoritics and Planetary Science 39, A99

Swindle, T.D. \& Kring D.A. 2001, in: Eleventh Annual V.M. Goldschmidt Conference, LPI Contribution No. 1088, Lunar and Planetary Institute, Abstract 3785

Walker, R.J., Horan M.F., Morgan J.W., Becker H., Grossman J.N., \& Rubin A.E. 2002, Geochim. Cosmochim. Acta 66, 4187

Watson, L.L., Hutcheon I.D., Epstein S., \& Stolper E.M. 1994, Science 265, 86

Watson, E.B. \& Harrison, T.M. 2005, Science 308, 841

Weaver, H.A., Feldman P.D., Combi M.R., Krasnopolsky V., Lisse, C.M., \& Shermansky, D.E. 2002, ApJ. 576, L95

Weirich, J.R., Brown R.H., \& Lauretta D.S. 2004, Bull. Amer. Astron Assoc. 36, 1143.

Wetherill, G.W. 1985, Science 228, 877

Wilde, S.A., Valley, J.W., Peck, W.H., \& Graham, C.M. 2001, Nature 409, 175

Zahnle, K.J. \& Sleep, N.H. 1997, in: P.J. Thomas, et al. (eds.) Comets and the Origin and Evolution of Life, (New York: Springer Velag), P. 194 